\title{
Oviposition by Indian Rock Pythons, Python molurus (Linnaeus 1758), in an Artificial Refuge
}

\author{
Jaydeep Maheta ${ }^{1}$, Khan Ashaharraza ${ }^{2}$, Vishal Varma ${ }^{3}$, Shital Dave ${ }^{4}$, Uday Mehta ${ }^{5}$, Rushi Pathak ${ }^{6}$, and Akshita Patel \\ ${ }^{1}$ Kajipur, Post Hansalpur (Shereswar), Viramgam, Ahmedabad, Gujarat, India \\ ${ }^{2}$ Department of Wildlife and Biodiversity Conservation, North Orissa University, Baripada, Orissa-757003, India \\ 3416, Bombay Natural History Society, Mumbai-400001, Maharashtra, India (vishal.varma789@gmail.com) \\ ${ }^{4}$ Department of Zoology, Maharaja Krishnkumar Sihnji University, Bhavnagar, Gujarat, India \\ ${ }^{5} 165$, Brahmanavas shilaj gaam, Ahmedabad, Gujarat, India \\ 65 Xitiji row house, Near City Gold Multiplex, Bopal road, Ambli, Ahmedabad, Gujarat, India \\ ${ }^{7}$ B.R. Doshi School of Bioscience, Anand, Gujarat, India
}

$\mathrm{R}^{2}$ amesh and Bhupathy (2010) reviewed the literature pertaining to the breeding biology of Indian Rock Pythons (Python molurus), much of which is based on observations in captivity. Herein we document an instance of two Indian Rock Pythons depositing and incubating eggs in an artificial refuge (Fig. 1).

At 1600 h on 7 July 2017, during a visit to Khengaria, Ahmadabad District, Gujarat, India $\left(23.014116^{\circ} \mathrm{N}\right.$, $72.043059^{\circ} \mathrm{E}$ ), we found two pythons, about $170 \mathrm{~cm}$ apart, coiled around egg clutches inside a concrete underground pipe measuring $25.4 \mathrm{~cm}$ in diameter (Fig. 2). The larger female nearest the opening of the pipe was approximately $2.6 \mathrm{~m}$ in total length and had a clutch of 14 eggs. The other female, with a total length of about $2.1 \mathrm{~m}$, had a clutch of 22 eggs. When we checked on the snakes a month later, both females had abandoned the site, but remains of egghells indicated that both clutches had hatched successfully.

\section{Acknowledgements}

We thank the Gujarat Forest Department, Wildlife Care Centre, Ahmedabad, and our team members, Arif Theba,

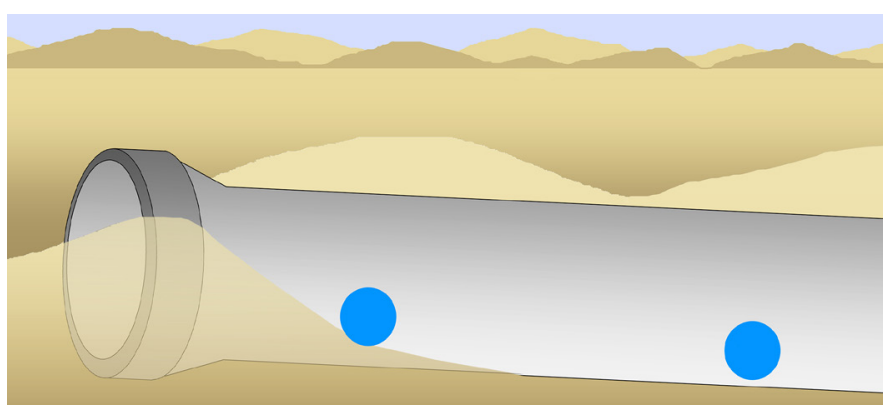

Fig. 2. Schematic of the concrete pipe showing the location of the two Indian Rock Pythons (Python molurus) and their clutches (indicated by blue circles). Illustration by Khan Ashaharraza.

Ami Shrimali, Gopal Jani, Sanjay Chavda, Rohit Sabaliya, Abhishek Jadwani, Devarshi Nayak, and Darshan Soni.

\section{Literature Cited}

Ramesh, C. and S. Bhupathy. 2010. Breeding biology of Python molurus molurus in Keoladeo National Park, Bharatpur, India. Herpetological Journal 20: 157-163.

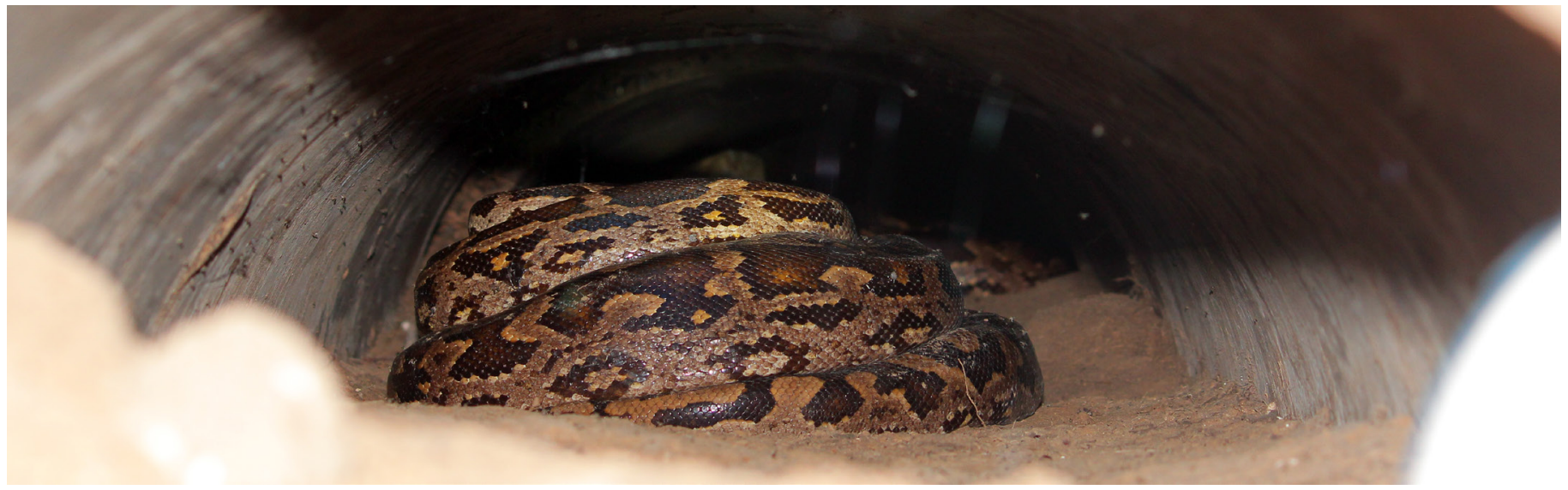

Fig. 1. Two Indian Rock Pythons (Python molurus) attending eggs inside a concrete pipe. Photograph by Jaydeep Maheta. 